\title{
Change of electronic properties on transition from high-entropy to Ni-rich $(\mathrm{TiZrNbCu})_{1-x} \mathrm{Ni}_{x}$ alloys
}

\author{
Marko Kuveždića ${ }^{\mathrm{a}}$, Emil Tafra ${ }^{\mathrm{a}}$, Mario Basletićc ${ }^{\mathrm{a}, *}$, Ramir Ristić ${ }^{\mathrm{b}}$, Petar \\ Pervan $^{\mathrm{c}}$, Vesna Mikšić Trontl ${ }^{\mathrm{c}}$, Ignacio A. Figueroa ${ }^{\mathrm{d}}$, Emil Babića \\ ${ }^{a}$ Department of Physics, Faculty of Science, University of Zagreb, Bijenička cesta 32, \\ 10000 Zagreb, Croatia \\ ${ }^{b}$ Department of Physics, University of Osijek, Trg Ljudevita Gaja 6, HR-31000 Osijek, \\ Croatia \\ ${ }^{c}$ Institute of Physics, Bijenička cesta 46, P. O. Box 304, HR-10001 Zagreb, Croatia \\ ${ }^{d}$ Institute for Materials Research-UNAM, Ciudad Universitaria Coyoacan, C.P. 04510 \\ Mexico D.F., Mexico
}

\begin{abstract}
We present results of comprehensive study of electronic properties of $(\mathrm{TiZrNbCu})_{1-x} \mathrm{Ni}_{x}$ metallic glasses performed in broad composition range $x$ encompassing both, high entropy (HE) range, and conventional Ni-base alloy concentration range, $x \geq 0.35$. The electronic structure studied by photoemission spectroscopy and low temperature specific heat (LTSH) reveal a split-band structure of density of states inside valence band with $d$-electrons of $\mathrm{Ti}, \mathrm{Zr}, \mathrm{Nb}$ and also Ni present at Fermi level $N\left(E_{F}\right)$, whereas LTSH and magnetoresistivity results show that variation of $N\left(E_{F}\right)$ with $x$ changes in Ni-base regime. The variation of superconducting transition temperatures with $x$ closely follows that of $N\left(E_{F}\right)$. The electrical resistivities of all alloys are high and decrease with increasing temperature over most of explored temperature range, and their temperature dependence seems dominated by weak localization effects over a broad temperature range $(10-300 \mathrm{~K})$. The preliminary study of Hall
\end{abstract}

\footnotetext{
${ }^{*}$ Corresponding author

Email address: basletic@phy.hr (Mario Basletić)
} 
effect shows positive Hall coefficient that decreases rapidly in Ni-base alloys. Keywords: metallic glasses, superconductors, electronic band structure, electronic properties, photoemission spectroscopy

\section{Introduction}

Recent concept of high entropy alloys (HEA, multicomponent near to equi-atomic alloys) [1, 2, 3, 4] has aroused large interest in the scientific community (see e.g., [5]) The concept of HEAs is in contrast to that of conventional alloys, which are commonly based on a single primary element that has been doped in order to promote a particular desired property. This alloy design explores the middle section of the phase diagrams of multicomponent alloys which was largely unexplored in conventional alloys [4]. Therefore, a virtually unlimited number of new alloys are now available for research and possible exploitation [6].

Intense research on HEAs over the last fifteen years has led to the production of several hundred new alloys [5] and publication of about two thousand papers, including several reviews of literature (e.g., [7, 8, 9, 10, 11, 12, 13, 14, 15]) and books [16, 17]. As a result, large progress has been made in the research and understanding of HEAs and several technologically relevant alloys, such as those with exceptional low- and high-temperature mechanical properties have been discovered [5].

However, present research of HEAs seems somewhat uneven, with the main effort invested in the development of new structural materials, thus focused on their mechanical properties, microstructure and thermal stability (e.g., [5, 14, 17]). Meanwhile, the research aimed at their electronic structures and properties, both experimental [5, 18, 19, 20, 21, 22, 23, 24] and 
theoretical [5, 25, 26, 27, 28, 29], is in spite of their potential as functional materials [5] still insufficient. The lack of insight into their electronic structure which in metallic systems determines almost all their properties (e.g., [30, 31]) hinders the conceptual understanding of both crystalline (c-) and amorphous (a-) HEAs [19, 24]. Furthermore, the distribution of research on HEAs in regards to their composition is highly uneven, with studies of HEAs based on 3d-metals forming a large majority, while there are only a few studies on HEAs composed of early and late transition metals (e.g., $[1,2,5,17,19,22,24,32])$. In general, in spite of their early appearance [1, 2] and large conceptual importance 17, 24, 32] research of a-HEAs lags well behind that of c-HEAs. This may be influenced by the fact that glass forming ability (GFA) of known a-HEAs is inferior in comparison to the best glass former in a particular alloy system [15, 17, 33]. However, in the last couple of years several comprehensive studies showing novel effects in a-HEAs have been published (e.g., [33, 34, 35, 36, 37, 38]).

The important problem of the transition from a-HEAs to conventional metallic glasses (MG) with the same chemical make-up was however ignored. The study of this transition is important both for understanding the formation of HEAs, and for proper evaluation of their potential in respect to that of conventional alloys.

Very recently, we reported, to our knowledge, the first systematic study of the atomic structure-electronic structure-property relationship in ( $\mathrm{TiZrNbCu})_{1-x} \mathrm{Ni}_{x}$ $(x \leq 0.5)$ MGs for $x$ in both the HEA $(x \leq 0.35)$ and conventional Ni-rich concentration range [22, 24]. It was found that both the atomic and the electronic structure show a change in the Ni-rich concentration range (valence electron number, $V E C \geq 7.4[22])$. This was reflected in all properties studied, including so-called boson peaks, by the change of their concentra- 
tion dependence for $x>0.35$. The results have been compared with those for corresponding binary and ternary MGs [24] sharing similar electronic structure.

Here we report the first, to the best of our knowledge, comprehensive study of the electronic properties of quinary MG system (TiZrNbCu) ${ }_{1-x} \mathrm{Ni}_{x}$ over a broad composition range $(x \leq 0.5)$ encompassing both the highentropy and the conventional alloy concentration range $(x>0.35)$. Electrical resistivities of all alloys are high $(\rho \geq 160 \mu \Omega \mathrm{cm})$ and show qualitatively the same variations with $x$ and temperature as in corresponding binary MGs [39, 40, 41, 42, 43, 44]. Superconducting transition temperatures $T_{c}$ decrease with $x$ as expected from the split-band electronic density of states revealed by ultraviolet photoemission spectroscopy (UPS). However, in agreement with the change of atomic and electronic structure on transition from aHEA to Ni-based MGs [24] the decrease of $T_{c}$ with $x$ declines for $x>0.35$. We also show that superconductivity in our alloys as well as that in crystalline HEAs [18, 21, 23] and binary transition metal alloys (e.g. [43]) is determined by their electronic structure and shows universal variation with the band (bare) density of states. The Hall coefficients, $R_{H}$, of all alloys are positive and decrease towards zero in Ni-rich alloys.

\section{Experimental}

As described in some detail elsewhere [19, 24], eight alloys in the (TiZrNbCu) ${ }_{1-x} \mathrm{Ni}_{x}$ system with $x=0,0.125,0.15,0.20,0.25,0.35,0.5$ and 0.55 were prepared from high purity elements by arc melting in a pure argon gas environment. Thin ribbons with thickness of about $20 \mu \mathrm{m}$ were fabricated from fragments of these alloys by melt spinning molten alloys on the surface of a copper roller 
in a pure He atmosphere [19, 22]. Detailed description of the methods employed for structural (X-ray powder diffraction, XRD), chemical (scanning electron microscopy with energy dispersive spectroscopy, SEM/EDS) and thermal (differential scanning calorimetry and thermogravimetric analysis, DSC-TGA) characterisation of all alloys was reported previously [19, 22, 24]. $\mathrm{XRD}$ and DSC results revealed that all samples except for those with $x=0$ and 0.55 were fully amorphous. Therefore, the samples with $x=0$ and 0.55 were not used in further studies. Details of the characterization of fully amorphous samples including their XRD patterns, SEM/EDS images and DSC curves were recently published [19, 22, 24]. We note that all these samples showed homogeneous distribution of elements with actual compositions close to nominal ones. The techniques employed for the measurements of their low temperature specific heat (LTSH), magnetic and mechanical properties were also reported recently [19, 22].

As described previously in [24], the valence-band structure of the as-cast sample was studied by UPS, with a Scienta SES100 hemispherical electron analyzer attached to an ultra-high vacuum chamber, with base pressure maintained below $10^{-9}$ mbar. An unpolarized photon beam of $21.2 \mathrm{eV}$ was generated by a He-discharge UV source. Several cycles of sputtering with $2 \mathrm{keV} \mathrm{Ar}^{+}$ions at room temperature were performed in order to remove oxygen and other contaminants from the sample surface. The energy resolution was about $25 \mathrm{meV}$.

The resistivity measurements were performed by the low frequency (typically $22 \mathrm{~Hz}$ ) ac method with rms current $i=0.2 \mathrm{~mA}$. About $8 \mathrm{~mm}$ long samples for resistivity measurements were mounted on the sample holder of a ${ }^{3} \mathrm{He}$ cryostat inserted into a 16/18 T Oxford superconducting magnet. The current and voltage wires were glued with silver paste onto the samples. 
The silver paste was allowed to dry at room temperature for about twelve hours resulting in contact resistances at most a few Ohms. The resistivity was determined from the measurements of resistance, length, mass and density of samples [44, 45, 46]. Due to finite width of the silver paste contacts the uncertainty in the absolute resistivity values was about 5\%. Additional uncertainty comes from voltage noise due to sizable Ag paste contact resistance. Compared to full voltage across the sample this noise was small (a few percents), i.e. less than the size of symbols in figures showing resistivities and superconducting transition. However, in a case of small signals such as the Hall voltage and magnetoresistance contributions (not shown) the noise was considerable part of the signal $(\simeq 10 \%)$ and is shown as the error bars. Raw UPS data are used so the error can be judged by the scatter in the intensity. As already stated the energy resolution of UPS was $25 \mathrm{meV}$, and is achieved by careful calibration of the setup. This uncertainty propagated into the values of dressed density of states $N\left(E_{F}\right)$ determined from the resistivity and upper critical field $H_{c 2}$ results. The measurements were performed in the temperature range $0.3-300 \mathrm{~K}$ in magnetic field $B$ perpendicular to the broad surface of the ribbon and to the current direction. The temperature was measured with a calibrated Cernox thermometer situated close to the samples. The Hall effect was measured on the same samples used for resistivity measurements, but with contacts arranged in the Hall geometry, at several temperatures between $1.4 \mathrm{~K}$ and $300 \mathrm{~K}$.

\section{Results and discussion}

As noted earlier [19, 22, 24], the values of thermophysical parameters such as the configurational entropy $\Delta S_{\text {conf }}$, the mixing $\Delta H_{\text {mix }}$ or formation 
enthalpy $\Delta H_{f}$ and the average difference in the atomic sizes of the constituents $\delta$, which are frequently employed in a semi-empirical criteria for the formation of different phases in HEAs [13], place (TiZrNbCu$)_{1-x} \mathrm{Ni}_{x}$ within the range of intermetallic compounds forming alloys. This is probably associated with strong interatomic interactions $\Delta H_{\text {mix }}<0$ and large atomic size difference $\delta$ between the early $(\mathrm{Ti}, \mathrm{Zr}, \mathrm{Nb})$ and late $(\mathrm{Ni}, \mathrm{Cu})$ transition metal components [24]. In this sense, these alloys can be viewed as quasi-binary alloys of early (TE) and late (TL) transition metals which will facilitate their comparison with conventional (binary and multicomponent) TE-TL alloys. Since a large (negative) $\Delta H_{\text {mix }}$ and $\delta$ also promote GFA in alloys [13, 19] the fabrication of amorphous alloys seems the simplest way to obtain $(\mathrm{TiZrNbCu})_{1-x} \mathrm{Ni}_{x}$ alloys in a single phase state. Indeed, careful characterization of our samples [19, 22, 24] revealed that alloys with $x=0.125,0.15,0.20,0.25,0.35$ and 0.5 were fully amorphous and that the distribution of the constituents within the samples was random. In what follows, we discuss only the results for fully amorphous, as-cast samples.

Figure 1 shows the variation of normalized resistivity with temperature for selected ( $\mathrm{TiZrNbCu})_{1-x} \mathrm{Ni}_{x}$ alloys. As is usual for both binary (e.g., [39, 40, 41, 42, 43, 44, 45, 46, 47, 48]) and multicomponent (e.g., [49]) TETL amorphous alloys with high resistivity $(\rho \geq 140 \mu \Omega \mathrm{cm})$ the resistivities of all our alloys decreased with increasing temperature above $4 \mathrm{~K}$. This correlation between the magnitude of electrical resistivity and the corresponding temperature coefficient of resistivity, $\mathrm{TCR}=d(\ln R) / d T$, at room temperature in concentrated disordered transition metal alloys was first observed by J. H. Mooij [50]. He attributed the transition from TCR $>0$ to TCR $<0$ at elevated resistivities to a small electronic mean free path, $l$, in a way similar to that used in Ioffe-Reggel criterion [51]. (This criterion 


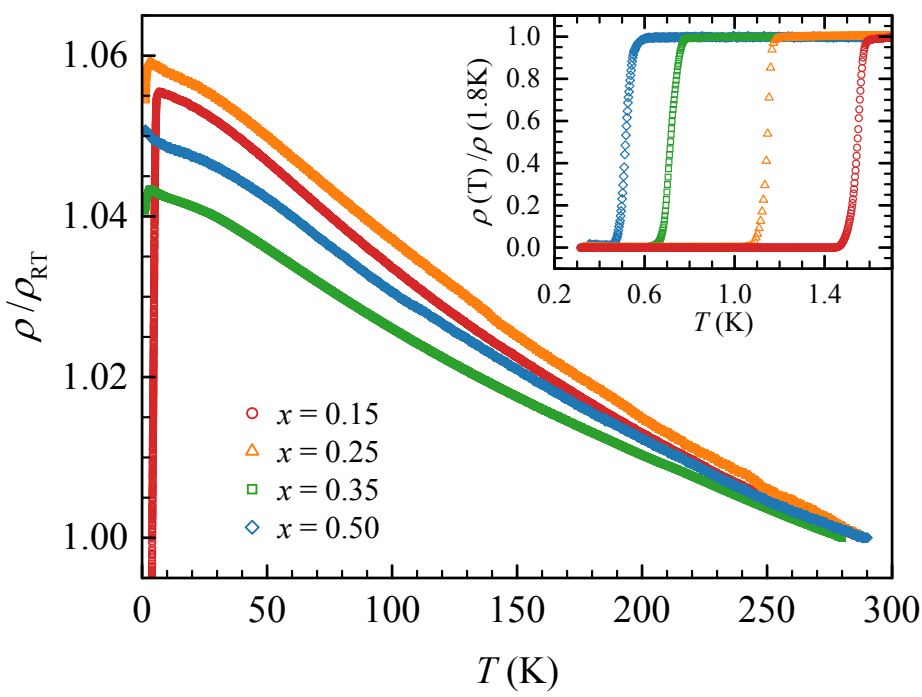

Figure 1: Normalized resistivities vs. temperature of selected ( $\mathrm{TiZrNbCu})_{1-x} \mathrm{Ni}_{x}$ amorphous alloys (Inset: superconducting resistivity transitions for the same alloys).

providing an estimate of the minimum metallic conductivity can be written in a form $k_{F} l \geq 1$, where $k_{F}$ is the Fermi wave-number which means that irrespective of the type of scattering, $l$ should not become smaller than the electronic wavelength. In other words, $k_{F} l<1$ is criterion for an onset of electron localization.) Both, the values of the resistivities and their semiconductor-like variations with temperature are long-standing problem and several models have been proposed for their explanation (e.g., [47, 48]). To the best of our knowledge the main mechanism(s) giving rise to the observed resistivities of amorphous TE-TL alloys has not been identified yet (e.g., [44, 47, 48]), whereas their temperature dependences seem to be dominated with the quantum interference effects (e.g. [40, 41, 42, 47]). The resistivities of our alloys, $166 \mu \Omega \mathrm{cm} \leq \rho \leq 176 \mu \Omega \mathrm{cm}$ at room temperature, 
showed within a sizable scatter, $\Delta \rho \approx \pm 10 \mu \Omega \mathrm{cm}$, caused by a finite width of silver paste voltage contacts, a tendency to increase a little with $x$ from $x=0.125$ to $x=0.35(\rho=176 \mu \Omega \mathrm{cm})$ and then decreased at $x=0.5$ $(\rho=170 \mu \Omega \mathrm{cm})$. Such values of resistivities and their variation with $x$ are typical for binary $\mathrm{TE}_{1-x} \mathrm{TL}_{x}$ amorphous alloys [39, 40, 41, 42, 47, 48] and were found to be insensitive on a type of TL for TL $=\mathrm{Cu}$ or Ni. Moreover, some other properties of $\mathrm{TE}_{1-x} \mathrm{TL}_{x}$ alloys such as the Debye temperature $\left(\Theta_{D}\right)$, Youngs modulus and even the superconducting transition temperature $T_{c}$, are quite insensitive to the replacement of $\mathrm{Ni}$ with $\mathrm{Cu}$. Because of this, in order to simplify the comparison of the results for our alloys with those for $\mathrm{TE}_{1-x} \mathrm{TL}_{x}$ alloys [24] it is convenient to express the stoichiometry of our alloys in a form $(\mathrm{TiZrNb})_{\left(1-x_{\mathrm{TL}}\right)}(\mathrm{TL})_{\left(x_{\mathrm{TL}}\right)}$ with total TL content $x_{\mathrm{TL}}=x(\mathrm{Cu}+\mathrm{Ni})=x+(1-x) / 4$, thus $0.344 \leq x_{\mathrm{TL}} \leq 0.625$ for studied alloys.

Figure 2 shows the variations of electrical conductivity $\sigma=\rho^{-1}$ with temperature for the alloys from HEA $(x=0.25)$ and Ni-rich $(x=0.5)$ concentration range. $\sigma$ for both alloys shows asymptotic $T$ and $T^{0.5}$ (upper abscissa) variations for temperatures below and above about $100 \mathrm{~K}$ respectively. These variations of $\sigma$ probably reflect the temperature dependence of the inelastic scattering time of electrons in non-magnetic alloys [40, 41, 42, 47]: $\tau_{i}^{-1} \sim T^{2}$ at lower temperatures and $\tau_{i}^{-1} \sim T$ for $T>100 \mathrm{~K}$ and follow from the expression for the electrical conductivity of three-dimensional disordered system in the regime of weak electron localization in the absence of magnetic field [52]:

$$
\Delta \sigma(H=0, T)=A(2 \sqrt{1+t}-\sqrt{t})
$$

where $A=\left(\sqrt{3} e^{2} / 2 \pi l\right) \sqrt{\tau / \tau_{\mathrm{so}}}, t=\tau_{\mathrm{so}} / 4 \tau_{i}$, and $\tau$ and $\tau_{\mathrm{so}}$ are electronic 


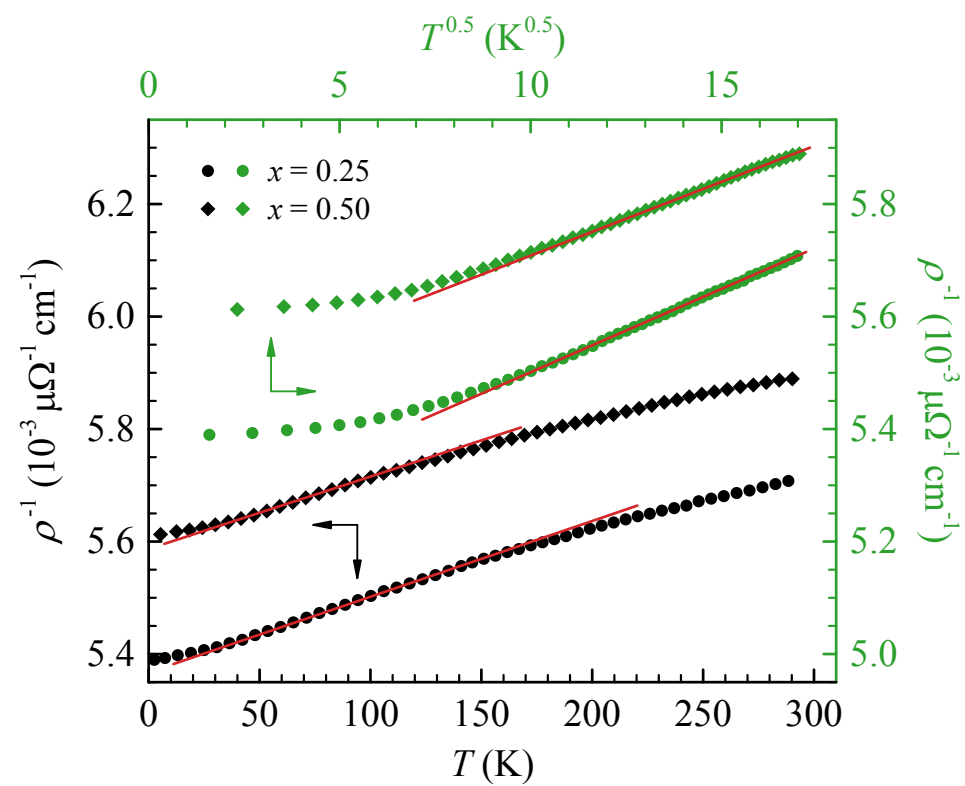

Figure 2: Left and bottom scale: $\rho^{-1}$ vs. $T$ for $(\mathrm{TiZrNbCu})_{1-x} \mathrm{Ni}_{x}$ alloys with $x=0.25$ and 0.5. (Lines denote $\rho^{-1} \propto T$ dependence.) Top and right scale: $\rho^{-1}$ vs. $T^{0.5}$ for the same alloys. (Lines denote $\rho^{-1} \propto T^{1 / 2}$ dependence.) 
transport life time and the life time due to spin orbit interaction, respectively. The complete expression for $\sigma(T)$ in weakly localized regime [52] includes in addition to delocalizing inelastic electron scattering $\tau_{i}$, and the effects of spin-orbit interaction $\tau_{\text {so }}$, and also the effects of the electron-electron $(e-e)$ interaction enhanced by disorder. We have shown earlier [42] that this expression provides a good, quantitative fit to experimental $\sigma(T)$ results for amorphous $\mathrm{Zr}_{42} \mathrm{Cu}_{58}$ alloy over a full explored temperature range, $4.2-150 \mathrm{~K}$. The effects of $\tau_{\mathrm{so}}$ and $(e-e)$ interaction dominate at the lowest temperatures and cause the upward deviation of $\sigma(T)$ from linearity (lower part of figure 2 and [42]). However, a self-consistent determination of both $\tau_{i}$ and $\tau_{\text {so }}$ requires the accurate measurements of the magnetoresistance at low temperatures. Our preliminary measurements of the low temperature magnetoresistance (MR) of the sample with $x=0.125$ showed qualitatively the behaviour which was predicted (Fig. 1 in 52 ) in case of weak localization and was experimentally observed (e.g. [47, 53]): rapid increase of MR at lower fields $\mu_{0} H$, followed by saturation at elevated fields. Such variation of MR with $\mu_{0} H$ is very different from simple $\left(\mu_{0} H\right)^{2}$ variation arising from the classical Lorentz force contribution (e.g. [54]). Unfortunately, rather large scatter of our experimental MR data (probably caused by the sizable resistance of the silver paste contacts and the effects of elevated fields) prevented their detailed analysis. Still, qualitatively the same variation of $\sigma(T)$ and $\mathrm{MR}$ observed in our and other $\mathrm{TE}_{1-x} \mathrm{TL}_{x}$ amorphous alloys indicates that the effects of incipient localization dominate the temperature dependence of resistivity in all these alloys. Some arguments [40, 41, 42] in support of this claim are: (1) very short electronic mean free paths, (2) a broad composition range showing a semiconducting resistivity behaviour, (3) direct relationship between the magnitude of increase of $\sigma$ with $T$ and 
electron-phonon interaction (figures 1 and 2 and [40, 41, 42]), (4) large lowtemperature magnetoresistance which cannot be accounted for with classical Lorentz force contribution, etc. All these arguments apparently apply to our alloys.

As illustrated in the inset to Figure 1 all samples were superconducting with transition temperatures $T_{c} \leq 1.6 \mathrm{~K}$. The resistive superconducting transitions were sharp, $\Delta T_{c} \leq 0.07 \mathrm{~K}$, where $\Delta T_{c}$ is the temperature interval between 0.1 and $0.9 R(1.8 \mathrm{~K})$. These sharp transitions, much sharper than those observed in Zr-based bulk metallic glasses [49], probably confirm good quality of our samples in accord with the results of XRD and SEM/EDS studies [19, 22]. As already noted [19], such low $T_{c} \mathrm{~s}$, comparable to these in amorphous $\mathrm{Hf}_{1-x} \mathrm{Cu}_{x}$ alloys [43], are unusual considering rather high contents of $\mathrm{Nb}$ (pure $\mathrm{Nb}$ has $T_{c}=9.2 \mathrm{~K}$ ), $\mathrm{Zr}$ and $\mathrm{Ti}$ which form alloys with $T_{c} \mathrm{~s}$ around $10 \mathrm{~K}$, but are consistent with the results for amorphous $\mathrm{Nb}-\mathrm{Zr}$ films which showed no superconductivity down to $T=1 \mathrm{~K}$. The monotonic decrease of $T_{c}$ with $x$ in our and all other $\mathrm{TE}_{1-x} \mathrm{TL}_{x}$ amorphous alloys (e.g., [43, 45, 46]) can be simply explained in terms of their electronic structures.

Figure 3 shows the UPS spectra for $(\mathrm{TiZrNbCu})_{1-x} \mathrm{Ni}_{x}$ alloys with $x=$ $0.125,0.2$ and 0.5 . These spectra reflect the evolution of the electronic density of states (DOS) within the valence band on increasing $\mathrm{Ni}$ content. All three spectra show clearly split-band structure of valence band with $\mathrm{Cu}-3 d$ states and Ni-3d states giving rise to spectral maxima at $3.5 \mathrm{eV}$ and $1.7 \mathrm{eV}$ below the Fermi level $\left(E_{F}\right)$. The spectral intensities at and close to $E_{F}$ are dominated with $d$-states of TEs; Ti, Zr and probably to some lower extent of $\mathrm{Nb}$ [24]. The $\mathrm{Ni}$ contribution to the intensity at $E_{F}$ is not negligible however and probably increases with increasing $x$. However, spectrum for $x=0.5\left(x_{\mathrm{TL}}=0.625\right)$ does not indicate band-crossing which 


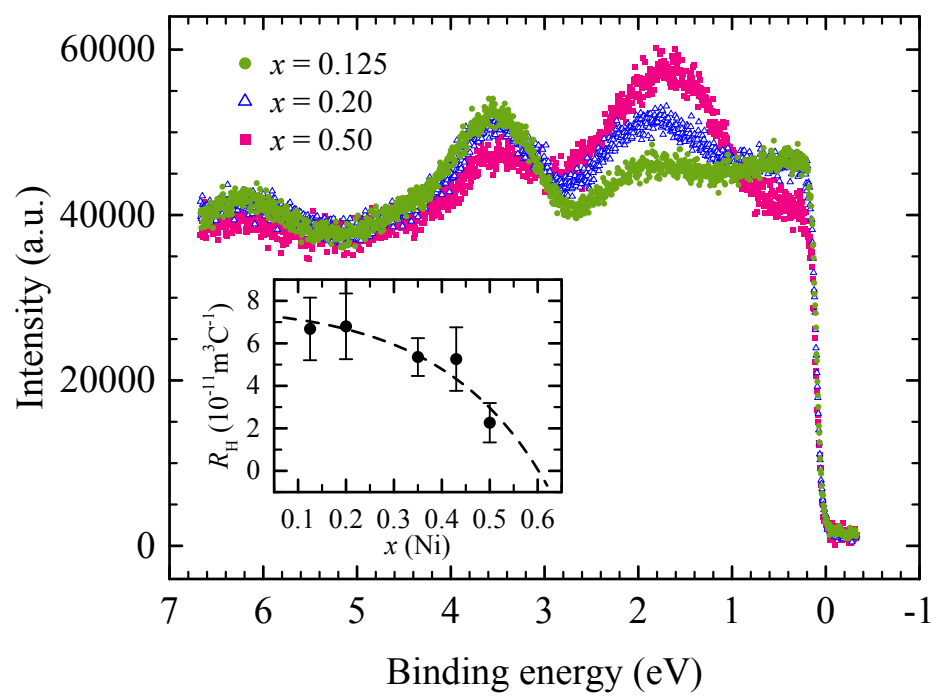

Figure 3: UPS spectra for ( $\mathrm{TiZrNbCu})_{1-x} \mathrm{Ni}_{x}$ samples with $x=0.125,0.2$ (data from [24]) and 0.5. Inset: Hall coefficients $R_{H}$ for $(\mathrm{TiZrNbCu})_{1-x} \mathrm{Ni}_{x}$ alloys vs. Ni concentration $x$. (Dashed line is a guide to the eye.) 
was observed in $\mathrm{Zr}_{1-x} \mathrm{Ni}_{x}$ alloys with $x \geq 0.64$ [24, 55, 56, 57]. In particular, $E_{F}$ of this alloy seems to remain in the area dominated with $d$-states of TEs. A more quantitative insight into the evolution of DOS at $E_{F}$ of our alloys with $x$ has been obtained recently from the LTSH measurements [22]. These measurements have shown that the coefficient $\gamma$ of the electronic contribution to LTSH, which is proportional to dressed DOS at $E_{F}, N_{\gamma}\left(E_{F}\right)$ $\left(\gamma=\pi^{2} k_{B}^{2} N_{\gamma}\left(E_{F}\right) / 3\right)$, where $k_{B}$ is the Boltzmann constant and $N_{\gamma}\left(E_{F}\right)=$ $N_{0}\left(E_{F}\right)\left(1+\lambda_{e-p}\right)$ where $\lambda_{e-p}$ represents the electron-phonon enhancement of DOS at $E_{F}, N_{0}\left(E_{F}\right)$, changes its variation with $x$ for $x>0.35$. In particular, $\gamma$ [22] and also $N_{\gamma}\left(E_{F}\right)$ (figure 4) decrease approximately linearly with $x$ for $x \leq 0.35$ (as expected from their split-band structure of DOS, figure 3) but seem to stop changing at $x=0.5$. The values of $\gamma$ for our alloys when plotted vs. $x_{\mathrm{TL}}$ nearly overlap those for binary amorphous $\mathrm{Zr}-\mathrm{Cu}$, Ni alloys [24] for $x_{\mathrm{TL}} \leq 0.513(x=0.35)$, but deviate strongly upwards from binary alloy data for $x_{\mathrm{TL}}=0.625(x=0.5)$. We note that the change in variation of $\gamma$ and $N_{\gamma}\left(E_{F}\right)$ with $x$ coincides with that in structural parameters of the same alloys occurring for $x_{\mathrm{TL}}>0.513$ (which corresponds to $V E C \geq 7.4$ ) [22, 24].

As seen in figure 4 the variation of $T_{c} \mathrm{~s}$ (defined as the midpoint of the resistive transitions in zero applied field, $0.51 \mathrm{~K} \leq T_{c} \leq 1.57 \mathrm{~K}$ ) of our alloys with $x$ and $V E C$ (upper abscissa) is qualitatively the same as that of $N_{\gamma}\left(E_{F}\right)$. (Probably better definition of $T_{c}$, free from the effects of a flux flow and superconducting fluctuations, would be as a temperature at which a deviation from the normal state behaviour appears, but in our measurements the scatter in the experimental resistivity data prevents an accurate determination of this temperature.) This indicates that superconductivity in $(\mathrm{TiZrNbCu})_{1-x} \mathrm{Ni}_{x}$ amorphous alloys irrespective of their composition and 


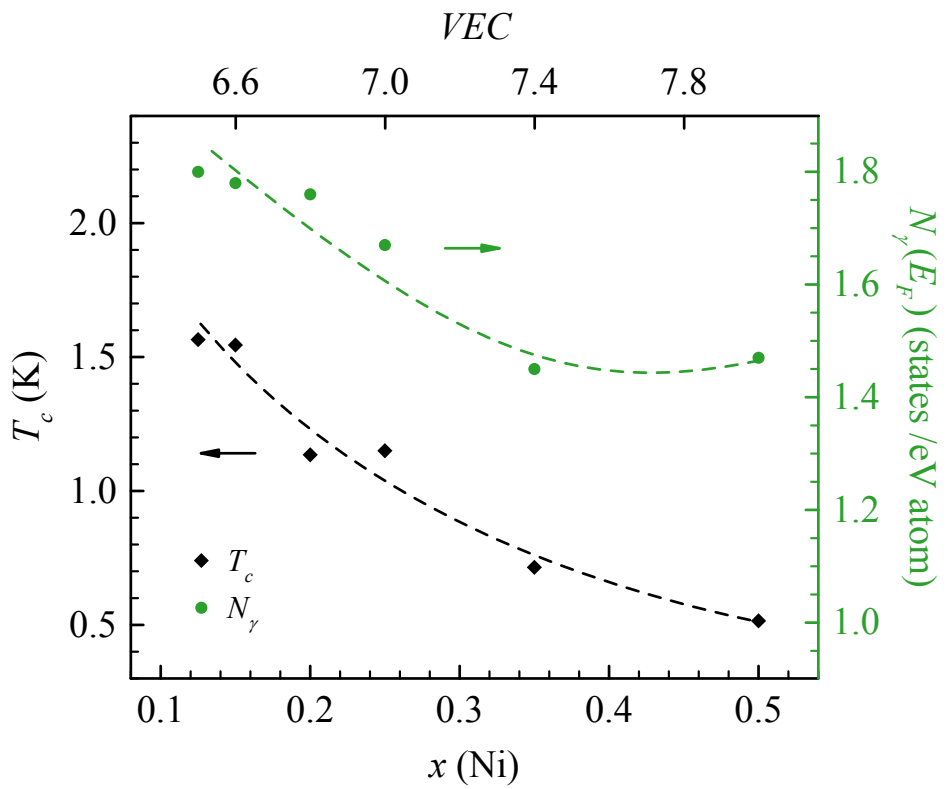

Figure 4: Left scale: $T_{c}$ vs. $x$, right scale: dressed density of states vs. $x$ for all $(\mathrm{TiZrNbCu})_{1-x} \mathrm{Ni}_{x}$ alloys (see text for details). Upper abscissa shows $V E C$. (Both dashed lines are a guides to the eye.) 
structural change obey the Dynes-Varma correlation [58] which seems valid for all disordered transition metal alloys, both crystalline (e.g., [21, 23]) and amorphous [43]. As already noted in [24], since $N_{\gamma}\left(E_{F}\right)$ is enhanced by the electron-phonon interaction, it cannot be used to prove that the changes in figure 4 for $x>0.35$ are due to change in $N_{0}\left(E_{F}\right)$. However, in superconducting transition metal alloys one can use the McMillan expression [59] in order to disentangle $N_{0}\left(E_{F}\right)$ from $\lambda_{e-p}$ in $N_{\gamma}\left(E_{F}\right)$. By using the data from figure 4 we calculated the values of $N_{0}\left(E_{F}\right)$ for all our alloys which confirmed a change in $N_{0}\left(E_{F}\right)$ for $x>0.35$. We also found that both, the values of $N_{0}\left(E_{F}\right)$ (ranging from 1.19 states $/ \mathrm{eV}$ atom for $x=0.125$ to 1.01 states/eV atom for $x=0.5$ ) and $\lambda_{e-p}$ (ranging from 0.51 for $x=0.125$ to 0.41 for $x=0.5$ ) of our alloys are somewhat lower than those for corresponding $\mathrm{Zr}-\mathrm{Cu}, \mathrm{Ni}$ amorphous alloys (e.g. [44]) which, together with the adverse influence of $\mathrm{Ti}$ [43, 58], probably explain their low $T_{C}$ s. Simultaneously, the initial rates of decrease of $T_{c}$ and $N_{\gamma}\left(E_{F}\right)$ with $x_{\mathrm{TL}}$ and $V E C$ of our alloys are very close to those in $\mathrm{Zr}_{1-x} \mathrm{Ni}_{x}$ alloys [45, 46] which implies that the same mechanism is responsible for the initial suppression of $T_{c}$ in both alloy systems.

Useful information about superconductivity in amorphous alloys can be obtained from the measurements of the upper critical field $H_{c 2}(T)$ (e.g. [43]). In particular, in the absence of LTSH results one can estimate $N_{\gamma}\left(E_{F}\right)$ from the measurements of $H_{c 2}(T)$ and $\rho$ :

$$
N_{\gamma}\left(E_{F}\right)=-\frac{\pi}{4 e k_{B} N_{A}} \frac{M}{\rho d}\left(\frac{\mathrm{d} H_{c 2}}{\mathrm{~d} T}\right)_{T=T_{c}}
$$

where $N_{A}, M$ and $d$, are the Avogadro number, molecular weight and mass density respectively, and other symbols have their usual meaning [43]. The $H_{c 2}(T)$ variations for selected $(\mathrm{TiZrNbCu})_{1-x} \mathrm{Ni}_{x}$ alloys encompassing both 


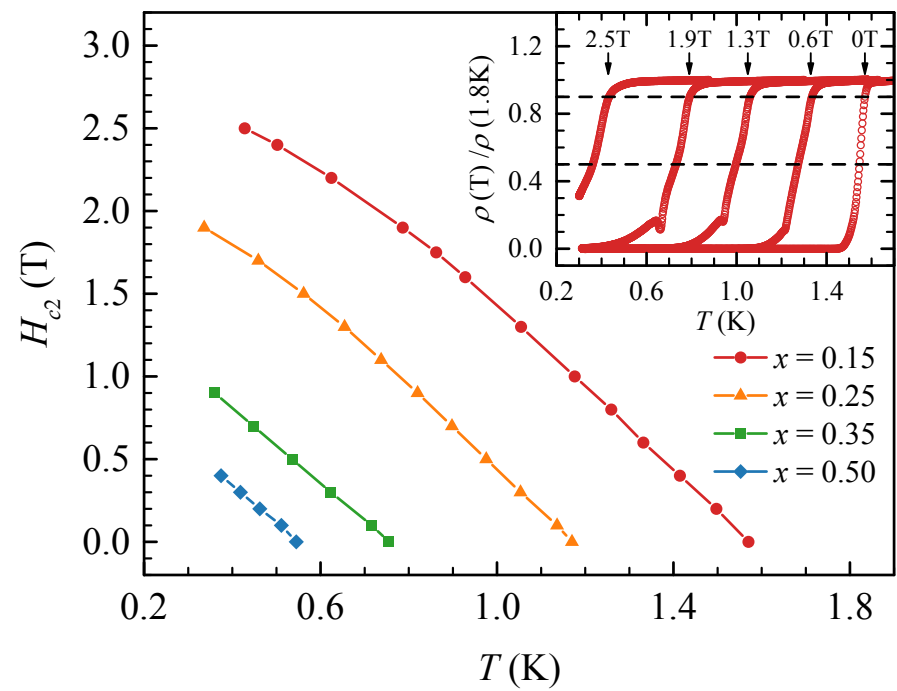

Figure 5: Upper critical fields $H_{c 2}(T)$ vs. $T$ for selected ( $\left.\mathrm{TiZrNbCu}\right)_{1-x} \mathrm{Ni}_{x}$ alloys. (Lines are a guides to the eye.) Inset: superconducting transition in alloy with $x=0.15$, for different fields.

HEA and Ni-base concentration range are shown in figure $5, H_{c 2}$ was defined with resistivity criterion $\rho\left(H_{c 2}\right)=0.9 \rho(1.8 \mathrm{~K})$, but as illustrated in the inset, due to sharp resistive transitions some other definitions of $H_{c 2}$ such as $0.5 \rho(1.8 \mathrm{~K})$ would cause very small downward shift of the data in figure 5. We note that magnetic field shifts and broadens the resistive transition but the broadening, caused by a weak flux pinning inherent to homogeneous amorphous superconductors, is the strongest for $\rho<0.5 \rho(1.8 \mathrm{~K})$ therefore its effect on the $H_{c 2}(T)$ variation is negligible.

The initial slopes of the upper critical field $\left(\mathrm{d} H_{c 2} / \mathrm{d} T\right)_{T_{c}}$ had values typical for amorphous $\mathrm{TE}_{1-x} \mathrm{TL}_{x}$ alloys $(2.3-2.6 \mathrm{~T} / \mathrm{K})$ and decreased with $x$, but this decrease stopped for $x>0.35$. By using (2) we have calculated $N_{\gamma}\left(E_{F}\right)$ from the corresponding results for $\left(\mathrm{d} H_{c 2} / \mathrm{d} T\right)_{T_{c}}$ and $\rho$. In spite of 
the rather large scatter, caused by the uncertainty in $\rho$, the calculated values of $N_{\gamma}\left(E_{F}\right)$ are close to those obtained from LTSH measurements (figure 4). In particular, these values of $N_{\gamma}\left(E_{F}\right)$ also showed a tendency to saturation for $x \geq 0.35$.

As is well known, $H_{c 2}$ also provides important information on the nature of superconductivity in the studied system [43]. In particular, $H_{c 2}$ provides the information on coherence length $\xi$, the fundamental length scale for superconducting state:

$$
\xi(T)=\sqrt{\frac{\Phi_{0}}{2 \pi B_{c 2}(T)}}
$$

where $\Phi_{0}=h / 2 e$ is the magnetic flux quantum. Further, $H_{c 2}$ provides important information on the mechanisms causing the destruction of superconductivity in applied magnetic field. However, due to low $T_{c} \mathrm{~s}$, the data for $H_{c 2}(T)$ of our samples does not cover a sufficiently broad range of reduced temperatures $t=T / T_{c}$ which is required in order to obtain reliable results to all parameters affecting $H_{c 2}(T)$ variation [43]. Because of this we focused on obtaining reliable estimates of $H_{c 2}(0)$ which provides an insight into the mechanism leading to the destruction of superconductivity. Accordingly, we performed fits of $H_{c 2}(t)$ data for samples with $x \leq 0.25$ for which both the explored $t$ range and the number of data points seem adequate (figure 5) for a reliable fitting. Since the fits of our data to the phenomenological expression $H_{c 2}(t)=H_{c 2}(0)\left(1-t^{2}\right)$ were not adequate we used a more general expression $H_{c 2}(t)=H_{c 2}(0)+B t+C t^{2}$ which provided good fit to experimental results. The obtained $H_{c 2}(0)$ values were close to Pauli limited field $H_{c 2}(0)=B_{\text {Pauli }} \approx 1.83 T_{c}$ where 1.83 comes from the BCS gap ratio, which indicates that depairing occurs when Zeman energy splitting becomes comparable to the energy gap. Thus, providing that $H_{c 2}(t)$ for Ni-based alloys 
also extrapolate close to $B_{\text {Pauli }}$ for $t \rightarrow 0$, the $H_{c 2}(0)$ values follow the same variation with $x$ as $T_{c}$ (figure (4). The values of $H_{c 2}(0)$ ranged from $2.9 \mathrm{~T}$ for $x=0.125$ to $0.94 \mathrm{~T}$ for $x=0.5$, with corresponding coherence length $107 \AA$ and $187 \AA$, respectively, see Eq. (3).

As already noted and shown explicitly in figure 6, our superconducting results obey quite well the Dynes-Varma correlations [58] for disordered transition metal alloys. According to this correlation the superconductivity in such alloys is determined with $N_{0}\left(E_{F}\right)$, which result in a linear variation of $\lambda_{e-p}$ with $N_{0}\left(E_{F}\right)$ with a slope that depends only on a class of transition metals (i.e., $3 d, 4 d$ or $5 d$ ) and the position of $E_{F}$ within a given $d$-band density of states [58]. As seen in figure [6, within the experimental scatter, our results accomodate quite well with the previous results for $5 d$-alloys [58], in spite of the fact that they do not contain $5 d$-metals. This probably arises from significant amount of $\mathrm{Ti}$ (3d-metal), which suppresses strongly the rate of increase of $\lambda_{e-p}$ with $N_{0}\left(E_{F}\right)$ as illustrated with the literature results for amorphous Ti-Ni alloys [57] in figure 6. Similarly, the results for crystalline (bcc) Ta-Nb-Zr-Hf-Ti HEAs (which contain components from all transition metal series) are extending between the lines drawn previously for $4 d$ and $5 d$-alloys [58] and their position depends on the fraction of $3 d$, $4 d$ and $5 d$ metal. In particular, alloy with lowest Ti content is closest to $4 d$ line, whereas these with highest Ta content are located at the $5 d$-line. We note that results for HEAs in figure [ extend the validity of Dynes-Varma correlation to mixtures of different transition metals.

The Hall coefficient of $(\mathrm{TiZrNbCu})_{1-x} \mathrm{Ni}_{x}$ alloys studied so far is positive and has the values similar to those observed in amorphous $\mathrm{Zr}-\mathrm{Co}, \mathrm{Ni}, \mathrm{Cu}$ alloys, $R_{H} \leq 6.8 \times 10^{-11} \mathrm{~m}^{3} \mathrm{C}^{-1}\left[55\right.$, ,56]. As seen in the inset to Fig. 3, $R_{H}$ is practically constant for $x \leq 0.2$ and decreases rapidly for $x \geq 0.43$, reaching 


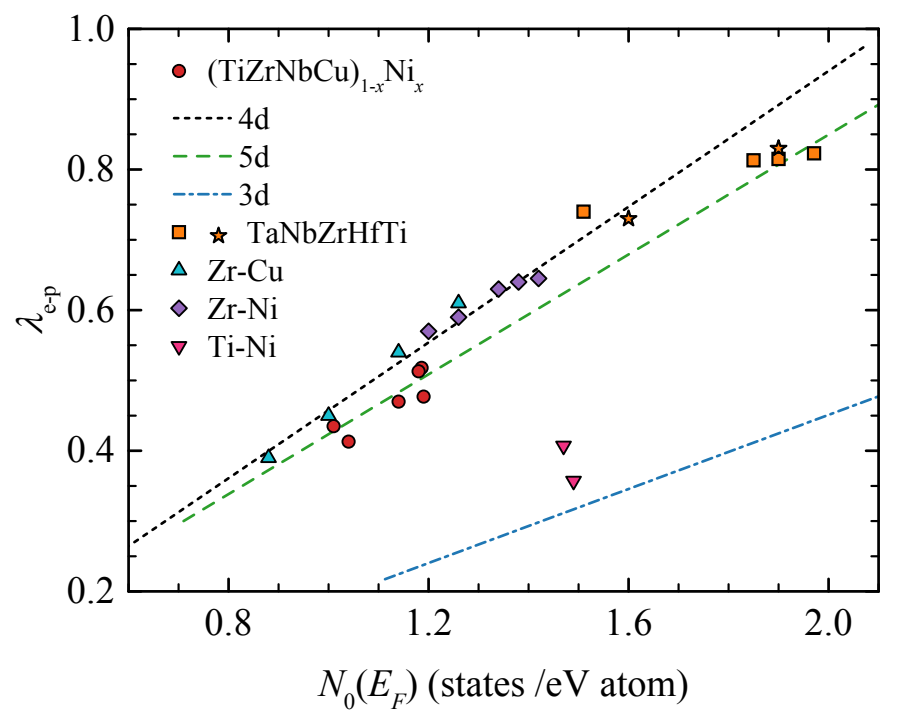

Figure 6: Electron-phonon coupling constant vs. band density of states at the Fermy level for disordered transition metals and their alloys. Circles represent our data; lines are from ref. [58]; squares are from [18, 211]; stars are from [23]; up-triangles are from [60]; diamonds are from [45, 61]; down-triangles are from [62]. (Dashed lines corresponds to prediction in Ref. [58].) 
$2.2 \times 10^{-11} \mathrm{~m}^{3} \mathrm{C}^{-1}$ for $x=0.5$. A similar decrease of $R_{H}$ in amorphous $\mathrm{Zr}-\mathrm{Ni}$, Co alloys has been associated with approach to crossing of $E_{F}$ from Zr $d$-band to the solute $d$-band around a certain concentration $x_{c}$. Linear extrapolation of the variation of $R_{H}$ between $x=0.35$ and $x=0.5$ to $R_{H}=0$ yielded $x_{c}=0.61$ which is consistent with the approximate expression for $x_{c}$ developed in [55, 56]. Thus, the variation of $R_{H}$ in multicomponent TE-TL amorphous alloys is, as in corresponding binary alloys [55, 56], determined by the changes in their electronic structure. However, the origin of $R_{H}>0$ in these alloys is still not completely understood [44].

\section{Conclusion}

Main results of the comprehensive study of electronic properties of $(\mathrm{TiZrNbCu})_{1-x} \mathrm{Ni}_{x}$ amorphous alloys over a broad composition range (encompassing both highentropy and conventional alloy concentration range $x>0.35$ ) are presented and compared with those for corresponding binary and multicomponent amorphous alloys of early (TE) and late (TL) transition metals.

The observed magnitudes of the electrical resistivities $(\rho)$ as well as their variations with $x$ and temperature $(T)$ are very similar to those in corresponding binary $\mathrm{TE}_{1-x} \mathrm{TL}_{x}$ alloys, which probably implies that chemical complexity has little effect on electronic transport in amorphous TE-TL alloys. In particular, both in binary and our quinary alloys the variations of $\rho$ with $T$ seem dominated with the quantum interference effects which show up in the magnetoresistance and specific variations of $\rho^{-1}$ vs. $T$ at lower and higher $(T \geq 100 \mathrm{~K})$ temperatures, respectively. The observed superconducting transition temperatures $T_{c}$ are surprisingly low $\left(T_{c} \leq 1.57 \mathrm{~K}\right)$ and decrease further with increasing $x$, as expected from their split-band 
electronic density of states with $d$-states of TEs dominant at the Fermi level $\left(E_{F}\right)$, as demonstrated by the photoemission spectra for $x=0.125,0.2$ and 0.5. The decrease of $T_{c}$ with total TL content $x_{\mathrm{TL}}$ and $V E C$ within the high-entropy concentration range were practically the same as in binary $\mathrm{Zr}_{1-x} \mathrm{Ni}_{x}$ alloys implying the same mechanism for the suppression of superconductivity. However, in agreement with the change in atomic and electronic structure on transition from HEA to Ni-based alloys, the decrease of $T_{c}$ with $x$ declined for $x>0.35$. Thus, $T_{c}$ is determined by the density of states at $E_{F}$ as is usual in disordered transition metal alloys. Moreover, both our and the literature results for crystalline HEAs show that superconductivity in alloys containing mixture of different transition metals is also determined with the band density of states. Since the estimated upper critical fields at $T=0 \mathrm{~K}\left(H_{c 2}(0)\right)$ seem to be Pauli limiting fields, the variation of $H_{c 2}(0)$ with $x$ is probably the same as that of $T_{c}$. The preliminary results for the Hall effect show that $R_{H}>0$ and has a similar magnitude as that in $\mathrm{Zr}_{1-x} \mathrm{TL}_{x}$ alloys with low $x$. However, $R_{H}$ shows a tendency to change a sign to $R_{H}<0$ for $x>0.5$, which probably indicates that the relationship between the $R_{H}>0$ and band-crossing is the same as in binary $\mathrm{Zr}_{1-x} \mathrm{TL}_{x}$ amorphous alloys.

Summarizing the above, we note that the electronic properties of all disordered alloys of early and late transition metals strongly depend on their electronic structure and quenched-in disorder, and are less sensitive on their chemical complexity, i.e. number of components. (Indeed, recent study of the atomic structure, electronic structure and magnetism in simi$\operatorname{lar}(\mathrm{TiZrNbNi})_{1-x} \mathrm{Cu}_{x}$ MGs [63] revealed an ideal solution behaviour similar to the observed in binary TE-Cu MGs [44].) Therefore, the change in their properties on transition from HE to conventional alloy concentration 
range depends primarily on the corresponding change in their electronic and atomic structure.

\section{Acknowledgments}

Our research was financially supported by the Josip Juraj Strossmayer University of Osijek, and by the University of Zagreb through funds for multipurpose institutional financing of scientific research. We also acknowledge the support of project CeNIKS co-financed by the Croatian Government and the European Union through the European Regional Development Fund - Competitiveness and Cohesion Operational Programme (Grant No. KK.01.1.1.02.0013). I. A. Figueroa acknowledges the financial support of UNAM-DGAPA-PAPIIT.

\section{References}

[1] L. Ma, L. Wang, T. Zhang, A. Inoue, Bulk Glass Formation of Ti-ZrHf-Cu-M (M=Fe, Co, Ni) Alloys, Mater. Trans. 43 (2) (2002) 277-280. doi:10.2320/matertrans.43.277.

[2] B. Cantor, K. B. Kim, P. J. Warren, Novel Multicomponent Amorphous Alloys, Mater. Sci. Forum 386-388 (2002) 27-32. doi:10.4028/www.scientific.net/MSF.386-388.27.

[3] J.-W. Yeh, S.-K. Chen, S.-J. Lin, J.-Y. Gan, T.-S. Chin, T.-T. Shun, C.-H. Tsau, S.-Y. Chang, Nanostructured High-Entropy Alloys with Multiple Principal Elements: Novel Alloy Design Concepts and Outcomes, Adv. Eng. Mater. 6 (5) (2004) 299-303. doi:10.1002/adem.200300567. 
[4] B. Cantor, I. T. H. Chang, P. Knight, A. J. B. Vincent, Microstructural development in equiatomic multicomponent alloys, Mater. Sci. Eng. A 375-377 (2004) 213-218. doi:10.1016/j.msea.2003.10.257.

[5] M. C. Gao, P. K. Liaw, D. B. Miracle (Eds.), Focus Issue: Fundamental Understanding and Applications of High-Entropy Alloys, no. 33(19), J. Mater. Res., 2018. doi:10.1557/jmr.2018.347.

[6] O. N. Senkov, J. D. Miller, D. B. Miracle, C. Woodward, Accelerated exploration of multi-principal element alloys with solid solution phases, Nat. Commun. 6 (1) (2015) 6529. doi:10.1038/ncomms7529.

[7] E. J. Pickering, N. G. Jones, High-entropy alloys: A critical assessment of their founding principles and future prospects, Int. Mater. Rev. 61 (3) (2016) 183-202. doi:10.1080/09506608.2016.1180020.

[8] J.-W. Yeh, Physical Metallurgy of High-Entropy Alloys, JOM 67 (10) (2015) 2254-2261. doi:10.1007/s11837-015-1583-5.

[9] M.-H. Tsai, Physical Properties of High Entropy Alloys, Entropy 15 (12) (2013) 5338-5345. doi:10.3390/e15125338.

[10] B. Cantor, Multicomponent and High Entropy Alloys, Entropy 16 (9) (2014) 4749-4768. doi:10.3390/e16094749.

[11] Y. F. Ye, Q. Wang, J. Lu, C. T. Liu, Y. Yang, High-entropy alloy: Challenges and prospects, Mater. Today 19 (6) (2016) 349-362. doi:10.1016/j.mattod.2015.11.026.

[12] Y. Zhang, T. T. Zuo, Z. Tang, M. C. Gao, K. A. Dahmen, P. K. Liaw, Z. P. Lu, Microstructures and properties of high-entropy alloys, Prog. Mater. Sci. 61 (2014) 1-93. doi:10.1016/j.pmatsci.2013.10.001. 
[13] S. Guo, Phase selection rules for cast high entropy alloys: An overview, Mater. Sci. Technol. 31 (10) (2015) 1223-1230. doi:10.1179/1743284715Y.0000000018.

[14] D. B. Miracle, O. N. Senkov, A critical review of high entropy alloys and related concepts, Acta Mater. 122 (2017) 448-511. doi:10.1016/j.actamat.2016.08.081.

[15] Z. Wang, S. Guo, C. T. Liu, Phase Selection in High-Entropy Alloys: From Nonequilibrium to Equilibrium, JOM 66 (10) (2014) 1966-1972, and references therein. doi:10.1007/s11837-014-0953-8.

[16] B. S. Murty, J.-W. Yeh, S. Ranganathan, High-Entropy Alloys, Butterworth-Heinemann, London, 2014.

[17] M. C. Gao, J.-W. Yeh, P. K. Liaw, Y. Zhang (Eds.), High-Entropy Alloys: Fundamentals and Applications, Springer, Cham, 2016. doi:10.1007/978-3-319-27013-5

[18] P. Koželj, S. Vrtnik, A. Jelen, S. Jazbec, Z. Jagličić, S. Maiti, M. Feuerbacher, W. Steurer, J. Dolinšek, Discovery of a Superconducting High-Entropy Alloy, Phys. Rev. Lett. 113 (10) (2014) 107001. doi:10.1103/PhysRevLett.113.107001

[19] K. Biljaković, G. Remenyi, I. A. Figueroa, R. Ristić, D. Pajić, A. Kuršumović, D. Starešinić, K. Zadro, E. Babić, Electronic structure and properties of $(\mathrm{TiZrNbCu})_{1-\mathrm{x}} \mathrm{Ni}_{\mathrm{x}}$ high entropy amorphous alloys, J. Alloys Compd. 695 (2017) 2661-2668. doi:10.1016/j.jallcom.2016.11.179. 
[20] J. Lužnik, P. Koželj, S. Vrtnik, A. Jelen, Z. Jagličić, A. Meden, M. Feuerbacher, J. Dolinšek, Complex magnetism of Ho-Dy-Y-Gd-Tb hexagonal high-entropy alloy, Phys. Rev. B 92 (22) (2015) 224201. doi:10.1103/PhysRevB.92.224201.

[21] S. Vrtnik, P. Koželj, A. Meden, S. Maiti, W. Steurer, M. Feuerbacher, J. Dolinšek, Superconductivity in thermally annealed Ta-NbHf-Zr-Ti high-entropy alloys, J. Alloys Compd. 695 (2017) 3530-3540. doi:10.1016/j·jallcom.2016.11.417.

[22] I. A. Figueroa, R. Ristić, A. Kuršumović, K. Biljaković, D. Starešinić, D. Pajić, G. Remenyi, E. Babić, Properties of $(\mathrm{TiZrNbCu})_{1-\mathrm{x}} \mathrm{Ni}_{\mathrm{x}}$ metallic glasses, J. Alloys Compd. 745 (2018) 455-459. doi:10.1016/j.jallcom.2018.02.169.

[23] F. von Rohr, M. J. Winiarski, J. Tao, T. Klimczuk, R. J. Cava, Effect of electron count and chemical complexity in the Ta-Nb-Hf-Zr-Ti highentropy alloy superconductor, Proc. Natl. Acad. Sci. 113 (46) (2016) E7144-E7150. doi:10.1073/pnas.1615926113.

[24] E. Babić, D. Pajić, K. Zadro, K. Biljaković, V. Mikšić Trontl, P. Pervan, D. Starešinić, I. A. Figueroa, A. Kuršumović, Š. Michalik, A. Lachová, G. Remenyi, R. Ristić, Structure property relationship in $(\mathrm{TiZrNbCu})_{1-\mathrm{x}} \mathrm{Ni}_{\mathrm{x}}$ metallic glasses, J. Mater. Res. 33 (19) (2018) 31703183. doi:10.1557/jmr.2018.168.

[25] Y. Zhang, G. M. Stocks, K. Jin, C. Lu, H. Bei, B. C. Sales, L. Wang, L. K. Béland, R. E. Stoller, G. D. Samolyuk, M. Caro, A. Caro, W. J. Weber, Influence of chemical disorder on energy dissipation and defect 
evolution in concentrated solid solution alloys, Nat. Commun. 6 (1) (2015) 8736. doi:10.1038/ncomms9736.

[26] T. Zuo, M. C. Gao, L. Ouyang, X. Yang, Y. Cheng, R. Feng, S. Chen, P. K. Liaw, J. A. Hawk, Y. Zhang, Tailoring magnetic behavior of CoFeMnNiX (X=Al, Cr, Ga, and $\mathrm{Sn})$ high entropy alloys by metal doping, Acta Mater. 130 (2017) 10-18. doi:10.1016/j.actamat.2017.03.013

[27] Z. Wu, M. C. Troparevsky, Y. F. Gao, J. R. Morris, G. M. Stocks, H. Bei, Phase stability, physical properties and strengthening mechanisms of concentrated solid solution alloys, Curr. Opin. Solid State Mater. Sci. 21 (5) (2017) 267-284. doi:10.1016/j.cossms.2017.07.001.

[28] S. Huang, E. Holmström, O. Eriksson, L. Vitos, Mapping the magnetic transition temperatures for medium- and high-entropy alloys, Intermetallics 95 (2018) 80-84. doi:10.1016/j.intermet.2018.01.016.

[29] M. C. Gao, P. Gao, J. A. Hawk, L. Ouyang, D. E. Alman, M. Widom, Computational modeling of high-entropy alloys: Structures, thermodynamics and elasticity, J. Mater. Res. 32 (19) (2017) 3627-3641. doi:10.1557/jmr.2017.366.

[30] R. Ristić, K. Zadro, D. Pajić, I. A. Figueroa, E. Babić, On the origin of bulk glass forming ability in Cu-Hf, Zr alloys, EPL 114 (1) (2016) 17006, and references therein. doi:10.1209/0295-5075/114/17006.

[31] E. Babić, R. Ristić, I. A. Figueroa, D. Pajić, Ž. Skoko, K. Zadro, Electronic structure and glass forming ability in early and late 
transition metal alloys, Philos. Mag. 98 (8) (2018) 693-709. doi:10.1080/14786435.2017.1415467.

[32] A. Cunliffe, J. Plummer, I. Figueroa, I. Todd, Glass formation in a high entropy alloy system by design, Intermetallics 23 (2012) 204-207. doi:10.1016/j.intermet.2011.12.006.

[33] K. S. N. Satish Idury, B. S. Murty, J. Bhatt, Identifying non-equiatomic high entropy bulk metallic glass formers through thermodynamic approach: A theoretical perspective, J. Non-Cryst. Solids 450 (2016) 164173. doi:10.1016/j.jnoncrysol.2016.08.002

[34] M. Yang, X. J. Liu, H. H. Ruan, Y. Wu, H. Wang, Z. P. Lu, High thermal stability and sluggish crystallization kinetics of highentropy bulk metallic glasses, J. Appl. Phys. 119 (24) (2016) 245112. doi:10.1063/1.4955060.

[35] P. Gong, S. Zhao, H. Ding, K. Yao, X. Wang, Nonisothermal crystallization kinetics, fragility and thermodynamics of $\mathrm{Ti}_{20} \mathrm{Zr}_{20} \mathrm{Cu}_{20} \mathrm{Ni}_{20} \mathrm{Be}_{20}$ high entropy bulk metallic glass, J. Mater. Res. 30 (18) (2015) 27722782. doi:10.1557/jmr.2015.253.

[36] T. Qi, Y. Li, A. Takeuchi, G. Xie, H. Miao, W. Zhang, Soft magnetic $\mathrm{Fe}_{25} \mathrm{Co}_{25} \mathrm{Ni}_{25}(\mathrm{~B}, \mathrm{Si})_{25}$ high entropy bulk metallic glasses, Intermetallics 66 (2015) 8-12. doi:10.1016/j.intermet.2015.06.015.

[37] F. Wang, A. Inoue, F. L. Kong, Y. Han, S. L. Zhu, E. Shalaan, F. Al-Marouki, Formation, thermal stability and mechanical properties of high entropy (Fe, Co,Ni,Cr,Mo)-B amorphous al- 
loys, J. Alloys Compd. 732 (2018) 637-645, and references therein. doi:10.1016/j.jallcom.2017.10.227.

[38] J. Huo, L. Huo, J. Li, H. Men, X. Wang, A. Inoue, C. Chang, J.Q. Wang, R.-W. Li, High-entropy bulk metallic glasses as promising magnetic refrigerants, J. Appl. Phys. 117 (7) (2015) 073902. doi:10.1063/1.4908286.

[39] R. Ristić, E. Babić, K. Šaub, M. Miljak, Electrical and magnetic properties of amorphous $\mathrm{Zr}_{100-\mathrm{x}} \mathrm{Cu}_{\mathrm{x}}$ alloys, Fizika 15 (4) (1983) 363-373.

[40] K. Šaub, E. Babić, R. Ristić, Quantum corrections to conductivity of glassy $\mathrm{Zr}_{100-\mathrm{x}} \mathrm{Cu}_{\mathrm{x}}$ alloys, Solid State Commun. 53 (3) (1985) 269-272. doi : 10.1016/0038-1098(85)90050-X.

[41] E. Babić, K. Šaub, Universal conductivity variation in glassy Zr-M alloys, Solid State Commun. 56 (1) (1985) 111-115. doi:10.1016/0038-1098(85)90545-9.

[42] R. Ristić, E. Babić, K. Šaub, Temperature and concentration dependence of the electrical resistivity of $\mathrm{Zr}-\mathrm{Cu}$ and $\mathrm{Zr-Ni}$ glassy alloys, Fizika 21 (Supplement 1) (1989) 216-219.

[43] E. Tafra, M. Basletić, R. Ristić, E. Babić, A. Hamzić, Enhanced superconductivity in Hf-base metallic glasses, J. Phys. Condens. Matter 20 (42) (2008) 425215. doi:10.1088/0953-8984/20/42/425215.

[44] R. Ristić, J. R. Cooper, K. Zadro, D. Pajić, J. Ivkov, E. Babić, Ideal solution behaviour of glassy $\mathrm{Cu}-\mathrm{Ti}, \mathrm{Zr}$, Hf alloys and properties of amorphous copper, J. Alloys Compd. 621 (2015) 136-145, and references therein. doi:10.1016/j.jallcom.2014.09.167. 
[45] E. Babić, R. Ristić, M. Miljak, M. G. Scott, G. Gregan, Superconductivity in zirconium-nickel glasses, Solid State Commun. 39 (1) (1981) 139-141. doi:10.1016/0038-1098(81)91064-4.

[46] E. Babić, R. Ristić, M. Miljak, M. G. Scott, Electronic and Magnetic Properties of Zr-3d Glasses, in: T. Masumoto, K. Suzuki (Eds.), Proc. 4th Int. Conf. on Rapidly Quenched Metals, Japan Institute of Metals, Sendai, Japan, 1982, pp. 1079-1082.

[47] M. A. Howson, B. L. Gallagher, The electron transport properties of metallic glasses, Phys. Rep. 170 (5) (1988) 265-324. doi:10.1016/0370-1573(88)90145-7.

[48] Ž. Marohnić, M. Guberović, E. Babić, G. J. Morgan, Induced conductivity anisotropy in glassy $\mathrm{Zr}_{1-\mathrm{x}} \mathrm{M}_{\mathrm{x}}$ alloys, J. Phys. F Met. Phys. 17 (5) (1987) 1123-1134. doi:10.1088/0305-4608/17/5/012.

[49] D. Okai, T. Fukami, T. Yamasaki, T. Zhang, A. Inoue, Temperature dependence of heat capacity and electrical resistivity of $\mathrm{Zr}$ based bulk glassy alloys, Mater. Sci. Eng. A 375-377 (2004) 364-367. doi:10.1016/j.msea.2003.10.148.

[50] J. H. Mooij, Electrical conduction in concentrated disordered transition metal alloys, Phys. Status Solidi (A) 17 (2) (1973) 521-530. doi:10.1002/pssa.2210170217.

[51] A. Ioffe, A. Regel, Non-crystalline, amorphous and liquid electronic semiconductors, Prog. Semicond 4 (89) (1960) 237-291.

[52] H. Fukuyama, K. Hoshino, Effect of Spin-Orbit Interaction on Magnetoresistance in the Weakly Localized Regime of Three-Dimensional 
Disordered Systems, J. Phys. Soc. Jpn. 50 (7) (1981) 2131-2132. doi:10.1143/JPSJ.50.2131.

[53] J. Bieri, A. Fert, G. Creuzet, J. Ousset, Magnetoresistance of amorphous CuZr: Weak localization in a three dimensional system, Solid State Communications 49 (9) (1984) $849 \quad$ - 852. doi:10.1016/0038-1098(84)90436-8.

[54] A. Hamzi, E. Babi, Magnetoresistivity of Dilute AlMn Alloys, Sol. State Commun. $21 \quad$ (6) (1977) 607-610. doi:10.1016/0038-1098(77)90044-8.

[55] J. Ivkov, E. Babić, R. L. Jacobs, Hall effect and electronic structure of glassy Zr 3d alloys, J. Phys. F Met. Phys. 14 (4) (1984) L53-L57. doi:10.1088/0305-4608/14/4/003.

[56] J. Ivkov, E. Babić, On the origin of the positive Hall coefficient in disordered TE-TL alloys, J. Phys. Condens. Matter 2 (16) (1990) 38913896. doi:10.1088/0953-8984/2/16/019.

[57] I. Bakonyi, Electronic properties and atomic structure of (Ti, Zr, Hf)(Ni, Cu) metallic glasses, J. Non-Cryst. Solids 180 (2-3) (1995) 131-150. doi:10.1016/0022-3093(94)00472-2.

[58] R. C. Dynes, C. M. Varma, Superconductivity and electronic density of states, J. Phys. F Met. Phys. 6 (7) (1976) L215-L219. doi: $10.1088 / 0305-4608 / 6 / 7 / 004$.

[59] W. L. McMillan, Transition Temperature of Strong-Coupled Superconductors, Phys. Rev. $167 \quad$ (2) (1968) 331-344. doi:10.1103/PhysRev.167.331. 
[60] P. Garoche, J. Bigot, Comparison between amorphous and crystalline phases of copper-zirconium alloys by specific-heat measurements, Phys. Rev. B 28 (12) (1983) 6886-6895. doi:10.1103/PhysRevB.28.6886.

[61] M. Matsuura, U. Mizutani, Low-temperature specific heat study of $\mathrm{Ni}_{100-\mathrm{x}} \mathrm{Zr}_{\mathrm{x}}(\mathrm{x}=30-80)$ metallic glasses, J. Phys. F Met. Phys. 16 (8) (1986) L183-L189. doi:10.1088/0305-4608/16/8/006.

[62] S. Kanemaki, M. Suzuki, Y. Yamada, U. Mizutani, Low temperature specific heat, magnetic susceptibility and electrical resistivity measurements in Ni-Ti metallic glasses, J. Phys. F Met. Phys. 18 (1) (1988) 105-112. doi:10.1088/0305-4608/18/1/012.

[63] R. Ristić, I. A. Figueroa, A. Lachová, v. Michalik, V. Miki Trontl, P. Pervan, K. Zadro, D. Pajić, E. Babić, Transition from high-entropy to $\mathrm{Cu}$-based $(\mathrm{TiZrNbNi})_{1-x} \mathrm{Cu}_{x}$ metallic glasses, Journal of Applied Physics 126 (15) (2019) 154105. doi:10.1063/1.5119373. 